\title{
WEB BASED CONFERENCE SYSTEM FOR INTELLIGENCE TELEPRESENCE ROBOT: A FRAMEWORK
}

\author{
Bayu Kanigoro, Widodo Budiharto, Jurike V. Moniaga and Muhsin Shodiq \\ Computer Science Program, School of Computer Science, Bina Nusantara University, Jakarta, Indonesia
}

Received 2013-09-23, Revised 2013-09-27; Accepted 2013-11-07

\begin{abstract}
Once an individual has access to the Internet, there is a wide variety of different methods of communication and information exchange over the network, one of them is telepresence robot. This study presents a web framework for web conference system of intelligent telepresence robot. The robot is controlled using web conference system from Google App Engine, so the manager/supervisor at office/industry can direct the robot to the intended person to start a discussion/inspection. We build a web application for controlling the multiclient telepresence robot. Experimental result shows the ability of robot to be controlled remotely and smoothly using our framework.
\end{abstract}

Keywords: Telepresence System, Robotic

\section{INTRODUCTION}

Remote management has become a trend in recent times because of increasing activity outside an office or a factory. This is a constraint when a manager do business trip at other city, while surveillance activities in the office or factory can not be dismissed. With this requirement the robot teleprecense becomes one alternative solution in bridging between the manager who does not present in the office and the employee.

The idea of a mobile telepresence robot comes from the inherent limitations imposed by traditional video conferencing systems, in which interaction is restricted in a meeting room only. Telepresence is a specialized form of teleoperation and telerobotics whereby the operator of a remote system is presented with workstation facilities capable of creating the illusion that is actually present at a remote, hazardous worksite (Cassinis and Rojas, 1993). Telepresence robots can be deployed in a wide range of application domains which are in the workplaces, public sectors, amd home use. In Telerobotics, Automation and Human Supervisory Control, Sheridan shows that progress in robotics depends not only on change in technology, but also on advances in humans relationships to machines (Sheridan, 1992). Human Supervisory Control has the potential to bring robotics out of the laboratory and into the difficult and messy world (Sheridan, 1992).

This study present Web Application as part of teleconferencing system architecture in The Nationwide University Network in Indonesia (NUNI) Telepresence robot to improve its ability of the robot which can serve as web conference system which based from (Trahanias et al., 2005; Cosgun et al., 2013; Budiharto et al., 2012a; 2012b; 2013) works. The feature of the robot is following a user and conducts a web conference at the same time (Budihal et al., 2011) which gives easiness to help a user to move along and do something else while the web conference is on. However teleprensence systems nowadays using two ways communication audio and video transmission to transmit this formal information (Cosgun et al., 2013).

\section{THE SYSTEM ARCHITECTURE}

The Nationwide University Network in Indonesia (NUNI) Telepresence robot is based on Arduino microcontroller with three omni-directional wheels (Budiharto et al., 2013).

Corresponding Author: Bayu Kanigoro, Computer Science Program, School of Computer Science, Bina Nusantara University, Jakarta, Indonesia 


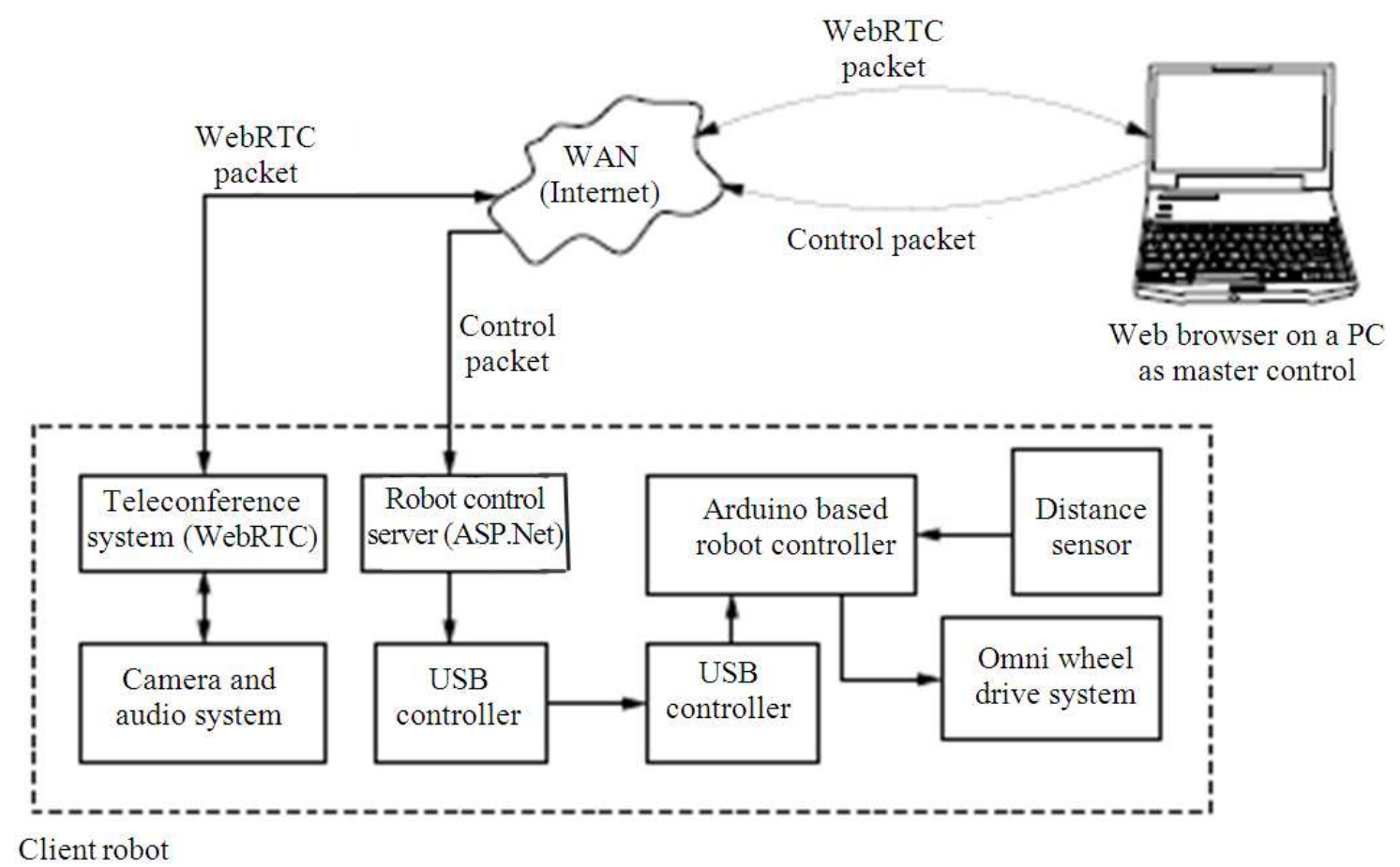

Fig. 1. NUNI Telepresence system architecture Budiharto et al. (2012a)

It has one meter aluminium arm mounted on its base plate and serves as elevated attachment point for telepresence system devices which are "14" monitor placed in a bracket, speakers, one camera for conversation and one stereo camera for people tracking, obstacles avoidance and can be used to extract additional features from environment. The robot has an overall height of $130 \mathrm{~cm}$, which allows both parties to communicate comfortably either by standing or sitting position. The system shown in Fig. 1 has two servers, Teleconference System and Robot Control Server, between Master Control and the robot. Teleconference System uses Google WebRTC as its service and ASP.Net is used for Robot Control Server to serve incoming control packet from Master Control. Figure 1 shows how camera and microphone send their image and sound streams through Internet and Master Control receives them. Other important part in Fig. 1 is Robot Control Server which gives capability of the robot which to be controlled from Master Control by sending messages to it. The parts messages consist of four instructions which are forward, backward, left and right. When the controller receives the messages from Master Controller through Internet, it passes through the messages to Arduino Based Robot Controller using USB interfaces. The Arduino Based Robot Controller translates the messages to Pulse Width
Modulation (PWM) then sends it to the motors. Besides the messages, the controller receives messages from distance sensor part shown in Fig. 1 to measure between the robot and an obstacle.

\section{THE DESIGN}

The use case of the web application is presented in Fig. 2. An user in Fig. 2 interacts with the system which has four event in it. The events are Send Control Message, Create Communication using Video and Audio, Connect to Telepresence Robot and Interact with Camera and Microphone. Send Control Message event is used to control the robot by clicking forward, backward, left and right button in the web application and convert the signal from the buttons to serial ASCII character which sent to robot controller. Second event is Create Communication of Video and Audio. This event create point-to-point connection by establishing a communication using Secure Real Time Protocol (SRTP) (Bergkvist et al., 2013) as transport medium for video and audio streams. A video stream uses VP8 codec (Bergkvist et al., 2013; Bankoski et al., 2011) and image enhancement to reduce the size of video without scarifying its quality then it can be transmitted through Internet with acceptable delay and loss. 


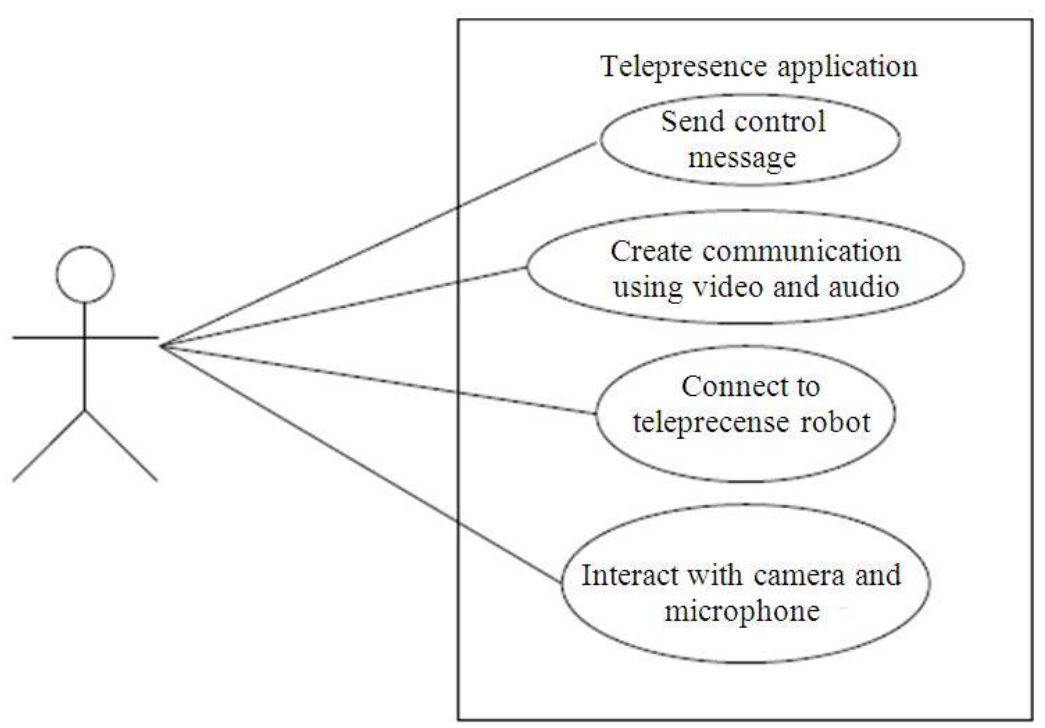

Fig. 2. Use case of the telepresence application

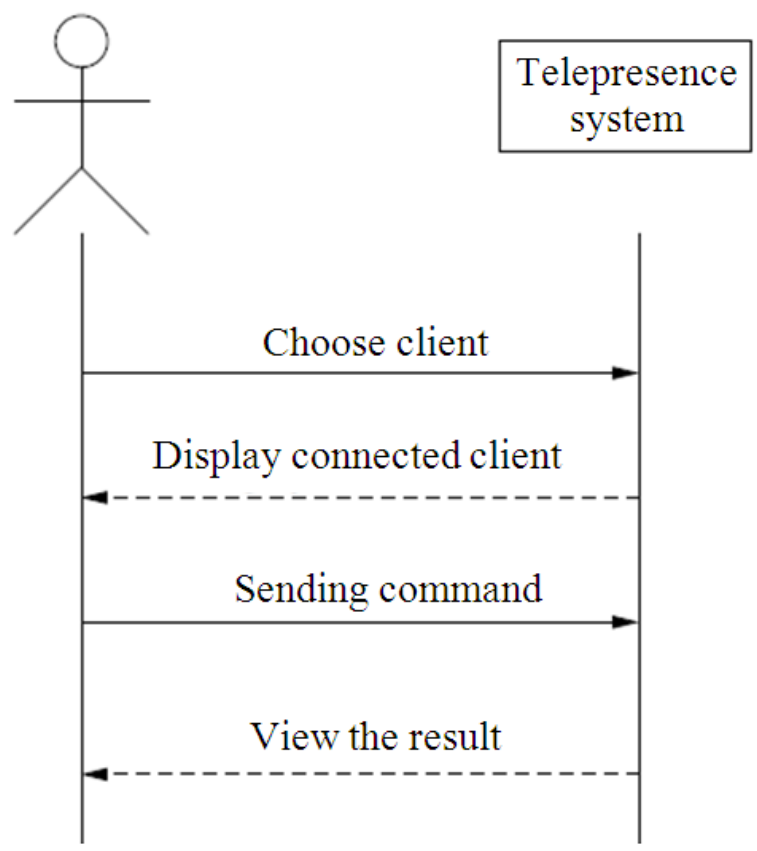

Fig. 3. Sequence diagram of the telepresence application

The other protocol named Internet Low Bit Rate Codec or iLBC protocol developed by Global IP Sound is employed when transmitting sound stream through Internet. The protocol designed for narrow band speech and uses payload bit rate around $13.33 \mathrm{kbit} / \mathrm{s}$ for $30 \mathrm{~ms}$ frames and $15.20 \mathrm{kbit} / \mathrm{s}$ for $20 \mathrm{~ms}$ frames (Hagen et al., 2004) which gives acceptable sound quality of telepresence system. Algorithm 1 is novel program to make the telepresence robot run smoothly while at the same time receiving command from the user under web application.

Figure 3 showed sequence diagram of the system. A user in Fig. 3 interacts with the system by choosing a client who want to be speak of then the system replied 
by displaying connected client to telepresence system. When a user wants to move the robot to its intended direction then it pushes a button in the web application to send a command shown in Fig. 3. Figure 4 shows activity diagram of web application which depicting how user interact with the Robot.

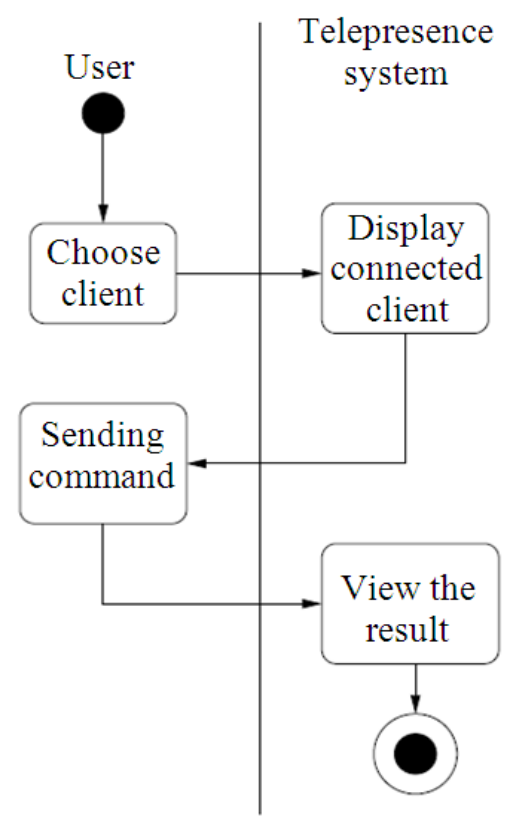

Fig. 4. Activity diagram of the telepresence application

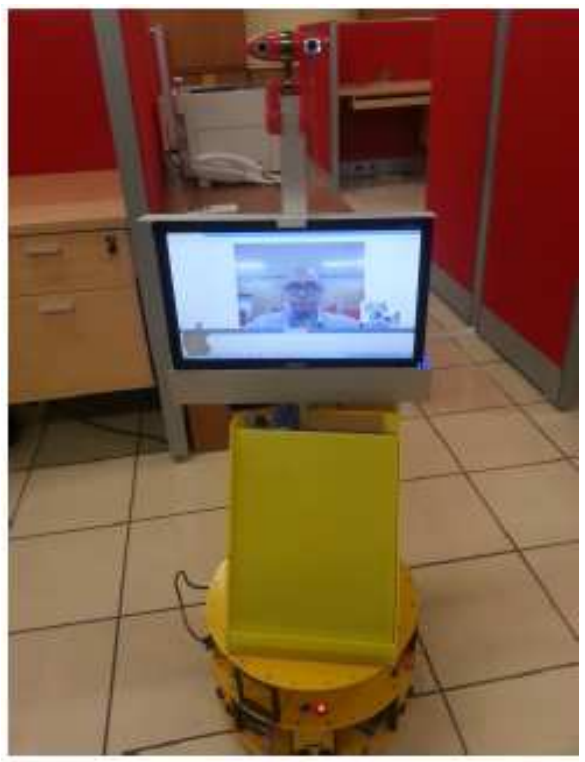

Fig. 5. The NUNI Telepresence robot

\section{RESULT}

Web application of The NUNI Telepresence robot has been developed using ASP.Net and WebRTC on Google App Engine for web based audio and video conferencing system which shows in Fig. 5. By employing WebRTC as implementation of SRTP protocol with VP8 and iLBC for video and audio codec respectively then it reduces delay, latency and packet payload through Internet. Button control shown in Fig. 6 is used to control movement of the robot. It has four buttons which have forward, reverse, right and left label then the robot will move to intended direction when one of four buttons is pushed. Initialization of the robot is conducted by runs the robot, server and teleconference system respectively then a link of web application which contains token number is sent to master control shown in Fig. 1 to be placed at address bar in WebRTC compatible browser.

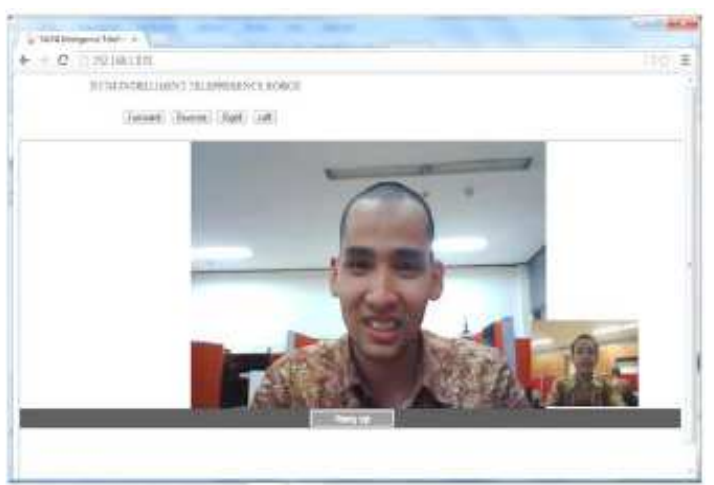

(a)

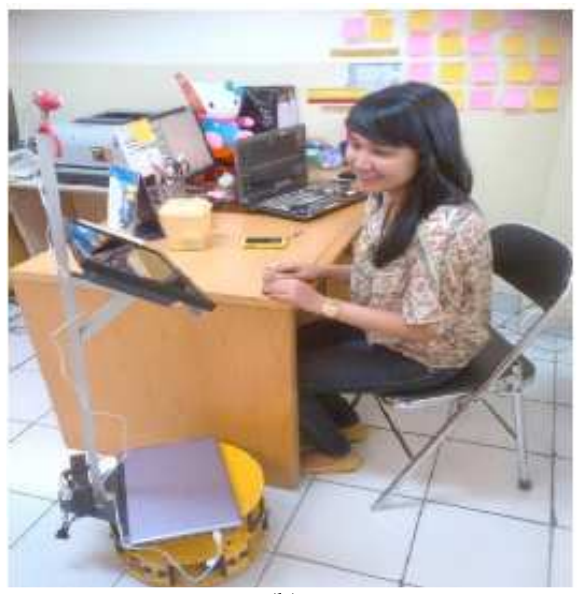

(b)

Fig. 6. The NUNI Telepresence robot runs web application to do a communication between employer and employee 


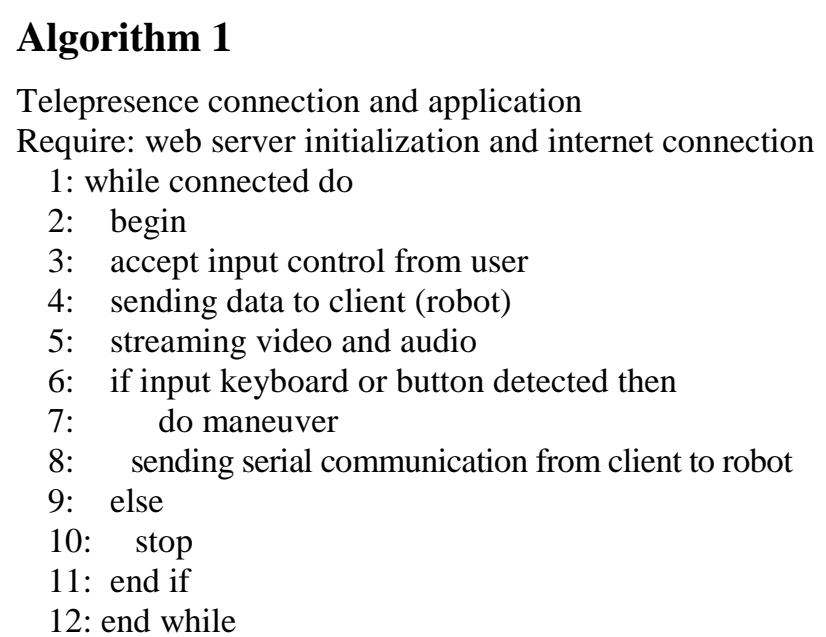

\section{CONCLUSION}

The paper presented a framework with UML for controlling telepresence robot using web application and its evaluation on a telepresence robot. The main contributions of this paper are a novel way of how conference conducted using Google App Engine and the insights about telepresence robots coming from the usability experiments.

The development of the web application in this study still manually controlled by a user by sending a command to the robot by pushing a button in the application which gives research challenge to develop an autonomous robot following an employer when moves instead of rests as suggested in (Cosgun et al., 2013).

\section{REFERENCES}

Bankoski, J., P. Wilkins and Y. Xu, 2011. Technical overview of vp8, an open source video codec for the web. Proceedings of the IEEE International Conference on Multimedia and Expo, Jul. 11-15, IEEE Xplore Press, Barcelona, pp: 1-6. DOI: 10.1109/ICME.2011.6012227

Bergkvist, A., D.C. Burnett, C. Jennings and A. Narayanan, 2013. Webrtc 1.0: Real-time communication between browsers. W3C Working Draft.
Budihal, R., N. Mohanan, S.A. Anand and S.S. Kamat, 2011. Exploration and implementation of a next generation telepresence system. Proceedings of the IEEE 5th International Conference on Advanced Networks and Telecommunication Systems, Dec. 18-21, IEEE Xplore Press, Bangalore, pp: 1-6. DOI: 10.1109/ANTS.2011.6163637

Budiharto, W., J. Moniaga, M. Meiliana and A. Aulia, 2013. A framework for obstacles avoidance of humanoid robot using stereo vision. Int. J. Adv. Robot. Syst., 10: 1-7. DOI: 10.5772/55608

Budiharto, W., M. Shodiq, B. Kanigoro and H. Hutomo, 2012a. The framework of intelligent Telepresence robot based on stereo vision. J. Comput. Sci., 8: 2062-2067. DOI: 10.3844/jcssp.2012.2062.2067

Budiharto, W., M. Shodiq, B. Kanigoro and J.V. Moniaga, 2012b. The framework of beebot: Binus multi-client of intelligent telepresence robot. World Acad. Sci., Eng. Technol., 71: 2010-2010.

Cassinis, R. and M.T. Rojas, 1993. "Intelligent" telepresence: Introducing virtual reality in advanced robots. Intell. Perceptual Syst., 745: 368-378. DOI: 10.1007/3-540-57379-8_23

Cosgun, A., D.A. Florencio and H.I. Christensen, 2013. Autonomous person following for Telepresence robots. Proceedings of the IEEE International Conference on Robotics and Automation, May 6-10, IEEE Xplore Press, Karlsruhe, Germany, pp: 43354342. DOI: 10.1109/ICRA.2013.6631191

Hagen, R., W. Kleijn, J. Linden and G.I. Sound, 2004. Internet low bit rate codec. Aalborg University.

Sheridan, T.B., 1992. Telerobotics, Automation and Human Supervisory Control. 1st Edn., MIT Press, Cambridge, ISBN-10: 0262193167, pp: 393.

Trahanias, P., W. Burgard, A. Argyros, D. Hahnel and H. Baltzakis et al., 2005. TOURBOT and WebFAIR: Web-operated mobile robots for tele-presence in populated exhibitions. Robot. Automat. Mag., 12: 77-89. DOI: 10.1109/MRA.2005.1458329 